\title{
Riqueza de borboletas em dois parques urbanos de Curitiba, Paraná, Brasil
}

\author{
Dayana Bonfantti, ${ }^{1,3}$, Luis Anderson Ribeiro Leite ${ }^{1}$, Marina Moraes Carlos ${ }^{1}$, \\ Mirna Martins Casagrande ${ }^{1}$, Érica Costa Mielke ${ }^{2}$ \& Olaf Hermann Hendrik Mielke ${ }^{1}$ \\ ${ }^{1}$ Departamento de Zoologia, Laboratório de Estudos de Lepidoptera Neotropical, \\ Universidade Federal do Paraná, CP 19020, CEP 81531-980, Curitiba, PR, Brasil \\ ${ }^{2}$ Secretaria Municipal do Meio Ambiente, Prefeitura Municipal de Curitiba, \\ Av. Manoel Ribas 2727, CEP 80810-100, Curitiba - PR, Brazil \\ ${ }^{3}$ Autor para correspondência: Dayana Bonfantti, e-mail: dayanabonfantti@gmail.com
}

BONFANTTI, D., LEITE, L.A.R., CARLOS, M.M., CASAGRANDE, M.M., MIELKE, E.C. \& MIELKE, O.H.H. Butterflies's richness in two urban parks in Curitiba Parana, Brazil. Biota Neotrop. 11(2): http://www. biotaneotropica.org.br/v11n2/en/abstract?inventory+bn00911022011

\begin{abstract}
In order to contribute to the knowledge of butterflies of Curitiba, were studied two urban municipal parks, the Botanical Garden which has total area of 178,000 $\mathrm{m}^{2}$ and has a grove of preserved Araucaria forest only for scientific research, and the Municipal Park of Barreirinha which has total area of 275,380 $\mathrm{m}^{2}$ and the area used for sampling of free access to population, with predominance of reforested vegetation. After 10 months of sampling, totaling 240 hours, 787 individuals and 166 species were registered, distributed to the families: Hesperiidae - 46 spp. (27.7\%), Nymphalidae - 75 spp. (45.2\%), Pieridae - 17 spp. (10.3\%), Lycaenidae - 13 spp. (7.8\%), Papilionidae -8 spp. (4.8\%) and Riodinidae - 7 spp. (4.2\%), among which 22 species are new records for Curitiba, belonging to the families: Hesperiidae, Pieridae, Lycaenidae, Riodinidae and Nymphalidae. About the recorded species, only $33 \%$ are considered common to the parks, Sorensen similarity index of $48 \%$ confirm that each location has a characteristic lepidopterofauna and distinct from each other.
\end{abstract}

Keywords: araucaria forest, butterflies, inventory.

BONFANTTI, D., LEITE, L.A.R., CARLOS, M.M., CASAGRANDE, M.M., MIELKE, E.C. \& MIELKE, O.H.H. Riqueza de borboletas em dois parques urbanos de Curitiba, Paraná, Brasil. Biota Neotrop. 11(2): http:// www.biotaneotropica.org.br/v11n2/pt/abstract?inventory+bn00911022011

Resumo: Com o objetivo de contribuir com o conhecimento das borboletas de Curitiba, foram estudados dois parques municipais urbanos, o Jardim Botânico que possui área total de $178.000 \mathrm{~m}^{2}$ e um bosque de Floresta Ombrófila Mista preservada restrito a pesquisa científica, e o Parque Municipal da Barreirinha que possui uma área total de $275.380 \mathrm{~m}^{2} \mathrm{e}$ a área utilizada para as amostragens de livre acesso a população, com predominância de vegetação reflorestada. Após 10 meses de amostragem, perfazendo 240 horas, foram registrados 787 indivíduos e 166 espécies, distribuídos nas famílias: Hesperiidae - 46 spp. (27,7\%), Nymphalidae - 75 spp. (45,2\%), Pieridae 17 spp. (10,3\%), Lycaenidae - 13 spp. (7,8\%), Papilionidae - 8 spp. (4,8\%) e Riodinidae - 7 spp. (4,2\%), dentre estas 22 espécies são novos registros para Curitiba, pertencentes as famílias: Hesperiidae, Pieridae, Lycaenidae, Riodinidae e Nymphalidae. Das espécies registradas, apenas 33\% são comuns a ambos os Parques, sendo que o índice de Similaridade de Sorensen de $48 \%$ confirma que cada um dos locais estudados possui lepidopterofauna característica e distinta um do outro.

Palavras-chave: floresta ombrófila mista, borboletas, inventário. 


\section{Introdução}

A ordem Lepidoptera está entre as maiores da classe Insecta, com cerca de 160.000 espécies descritas (Kristensen et al. 2007). Estimase que na região Neotropical encontram-se cerca de 8.000 espécies de borboletas (Lamas 2004), as quais podem ser utilizadas em pesquisas sobre biogeografia e interações inseto/planta, além de indicadoras em levantamentos, planejamento e administração de reservas naturais, devido à facilidade de encontrá-las e avaliá-las (Brown Junior 1992).

A primeira contribuição sobre a lepidopterofauna de Curitiba foi realizada por Bienzanko (1938) que catalogou 34 espécies de lepidópteros, das quais 24 espécies eram borboletas, em praças e parques municipais, assim como em campos vizinhos da cidade, entre os meses de maio a outubro de 1932. A segunda contribuição foi a de C. Mielke (1995) levantamento que se utilizou da Coleção do Departamento de Zoologia da Universidade Federal do Paraná, composta principalmente por coletas realizadas por O. H. H. Mielke e M. M. Casagrande, onde foram registradas 486 espécies.

O estudo das borboletas em áreas urbanas e a análise da estrutura e dinâmica destas populações em ambientes modificados já foi tema de diversos estudos (Ruszczyk 1986a,b, 1987; Brown Junior \& Freitas 2002; New \& Sands 2002; Shapiro 2002; Silva et al. 2007; Bonfantti et al. 2009). Para Ruszczyk (1986a), a ausência de vegetação nativa limita a distribuição de muitos lepidópteros, visto que há uma alta especificidade destes com recursos alimentares e plantas hospedeiras para suas larvas. Por essa razão a riqueza de vegetação nas áreas urbanas pode elevar a oferta de recursos e amenizar as condições adversas do meio urbano, atraindo novas populações de borboletas, assim áreas urbanas podem possuir fauna característica, distinta das demais.

O presente trabalho teve como objetivos realizar um inventário de borboletas em dois parques urbanos de Curitiba, o Jardim Botânico Municipal de Curitiba Francisca Maria Garfunkel Rischbieter e o Parque Municipal da Barreirinha, e comparar os resultados obtidos nestas áreas.

\section{Material e Métodos}

O município de Curitiba está localizado no primeiro planalto paranaense, com altitude média de $934 \mathrm{~m}$ acima do nível do mar, apresentando temperaturas máximas entre 23,7 a $32,5^{\circ} \mathrm{C}$ e mínimas de $-1,3$ a $15,9^{\circ} \mathrm{C}$, com média anual de $17,48^{\circ} \mathrm{C}$ (Instituto... 2010). O clima, no sistema de classificação de Köppen enquadra-se como Cfb, subtropical, úmido mesotérmico, de ventos fracos e com ocorrência de geadas severas, durante o inverno.

A cidade tem alto índice de áreas verdes, abrigando cerca de 30 parques e bosques. O Jardim Botânico Municipal de Curitiba Francisca Maria Garfunkel Rischbieter (2526’31' S e 49 14' 16" O) possui área total de $178.000 \mathrm{~m}^{2}$ e uma área de conservação, restrita a pesquisa científica, com um bosque de Floresta Ombrófila Mista preservada com aproximadamente $80.000 \mathrm{~m}^{2}$ (Figura 1). Constitui-se a única área verde próxima ao centro da cidade, localizado no Bairro Jardim Botânico entre a Rua Prefeito Lothário Meissner e Avenida Affonso Camargo, sendo estas duas vias de grande fluxo de veículos. O Parque Municipal da Barreirinha (2521'39' S e 49 $15^{\circ} 33^{\prime}$ ' O) situa-se no bairro Barreirinha, cerca de $9 \mathrm{Km}$ do centro da cidade, e é composto por áreas de lazer e lagos artificiais. Possui uma área total de $275.380 \mathrm{~m}^{2} \mathrm{e}$ a área utilizada para as amostragens possui cerca de $130.000 \mathrm{~m}^{2}$ e são de livre acesso a população (Figura 2). A vegetação é de Floresta Ombrófila Mista, embora tenha predominância de vegetação reflorestada, com dominância da planta invasora pauincenso, Pittosporum undulatum Vent. (Pittosporaceae) comumente utilizado para ornamentação.
As amostragens foram realizadas mensalmente entre agosto de 2008 a maio de 2009 para cada área de estudo, totalizando 10 meses. Estas foram realizadas com rede entomológica e armadilhas para borboletas frugívoras, utilizando-se como isca uma mistura de banana com caldo de cana. Uma caderneta de anotações foi utilizada para registrar a ocorrência de espécies já identificadas em amostragens anteriores. O esforço amostral para as observações foi das 9 horas às 15 horas, totalizando 12 horas de amostragem, com à presença de dois coletores. Devido às baixas temperaturas do inverno, as coletas foram interrompidas no mês de maio.

Com a finalidade de verificar a similaridade entre as áreas de estudo, foi aplicado o índice de similaridade de Sorensen, com valor representado em porcentagem.

O material coletado está depositado na Coleção Entomológica Padre Jesus Santiago Moure, Departamento de Zoologia da Universidade Federal do Paraná. Utilizou-se Lamas (2004) para a classificação das espécies e revisões de gêneros disponíveis na literatura.

\section{Resultados e Discussão}

Com 240 horas de amostragem, foram registrados 787 indivíduos de 166 espécies, assim distribuídos: Hesperiidae - 46 spp. (27,7\%), Nymphalidae - 75 spp. (45,2\%), Pieridae - 17 spp. (10,3\%), Lycaenidae - 13 spp. (7,8\%), Papilionidae - 8 spp. $(4,8 \%)$ e Riodinidae - 7 spp. (4,2\%). O presente estudo destaca 22 novos registros para Curitiba, pertencentes às famílias: Hesperiidae (10), Lycaenidae (7), Riodinidae (2) e Nymphalidae (3) (Tabela 1).

Para o Jardim Botânico foram registrados 392 indivíduos coletados/observados em 107 espécies, destas, 12 novos registros para Curitiba. Nymphalidae possuiu a maior riqueza $(49,53 \%)$, seguida por Hesperiidae $(22,43 \%)$, Pieridae $(12,15 \%)$, Lycaenidae (10,28\%), Papilionidae $(4,67 \%)$ e Riodinidae $(0,94 \%)$. O Parque Municipal da Barreirinha também foi amostrado em onze coletas, no qual foi possível registrar 395 indivíduos e 114 espécies, números mais altos apesar destes valores serem muito próximos aos registrados no Jardim Botânico. Foram encontrados oito novos registros para Curitiba, sendo que Rhetus periander eleusinus Stichel, 1901 e Adelpha falcipennis Fruhstorfer, 1915, são os registros novos comuns aos dois parques. Da mesma forma que o Jardim Botânico, Nymphalidae foi a família mais rica com $50 \%$ de representatividade, seguida por Hesperiidae $(25,44 \%)$, Pieridae $(8,77 \%)$, Riodinidae $(6,14 \%)$, Papilionidae $(5,26 \%)$ e Lycaenidae $(4,39 \%)$.

Dentre o total das espécies coletadas, $33 \%$ são comuns aos dois Parques. Da riqueza total do Parque Barreirinha 59 (52\%) são exclusivas, no Jardim Botânico estas são 53 (48\%). De acordo com o índice de Similaridade de Sorensen (Tabela 2), a semelhança na composição de espécies de borboletas entre os locais ficou abaixo de $50 \%$. A baixa similaridade também pode ser observada entre famílias, com destaque para Hesperiidae, Riodinidae e Lycaenidae que apresentaram respectivamente, 74, 75 e 63\% de dissimilaridade. Similaridade acima de $50 \%$ foi observada para as famílias, Nymphalidae $63 \%$, Papilionidae $55 \%$ e Pieridae $52 \%$.

Apesar de os Parques urbanos amostrados no presente estudo apresentarem valores de riqueza de espécies e abundância muito próximas, chama a atenção diferenças na composição da fauna de borboletas, evidenciando assembléias características de cada Parque. Possíveis razões para tal resultado podem estar associadas à conectividade dos locais estudados e a características gerais da fisionomia vegetal dos mesmos. O Jardim Botânico possui uma área de preservação e sua região de entorno é composta por edificações e rodovias, reduzindo assim a conectividade com outras áreas verdes (Figura 1). Por outro lado, o Parque Barreirinha apesar de estar 
Tabela 1. Lista de famílias, subfamílias, tribos e riqueza de espécies (S) capturadas em dois parques localizados na cidade de Curitiba, Parque Barreirinha (Bar) e Jardim Botânico (JBot). Novos registros de espécies na cidade de Curitiba (*).

Table 1. List of families, subfamilies, tribes and species (S) captured in two parks located in Curitiba, Municipal park of Barreirinha (Bar) and Botanical Garden (JBot). New records of species in Curitiba $(*)$.

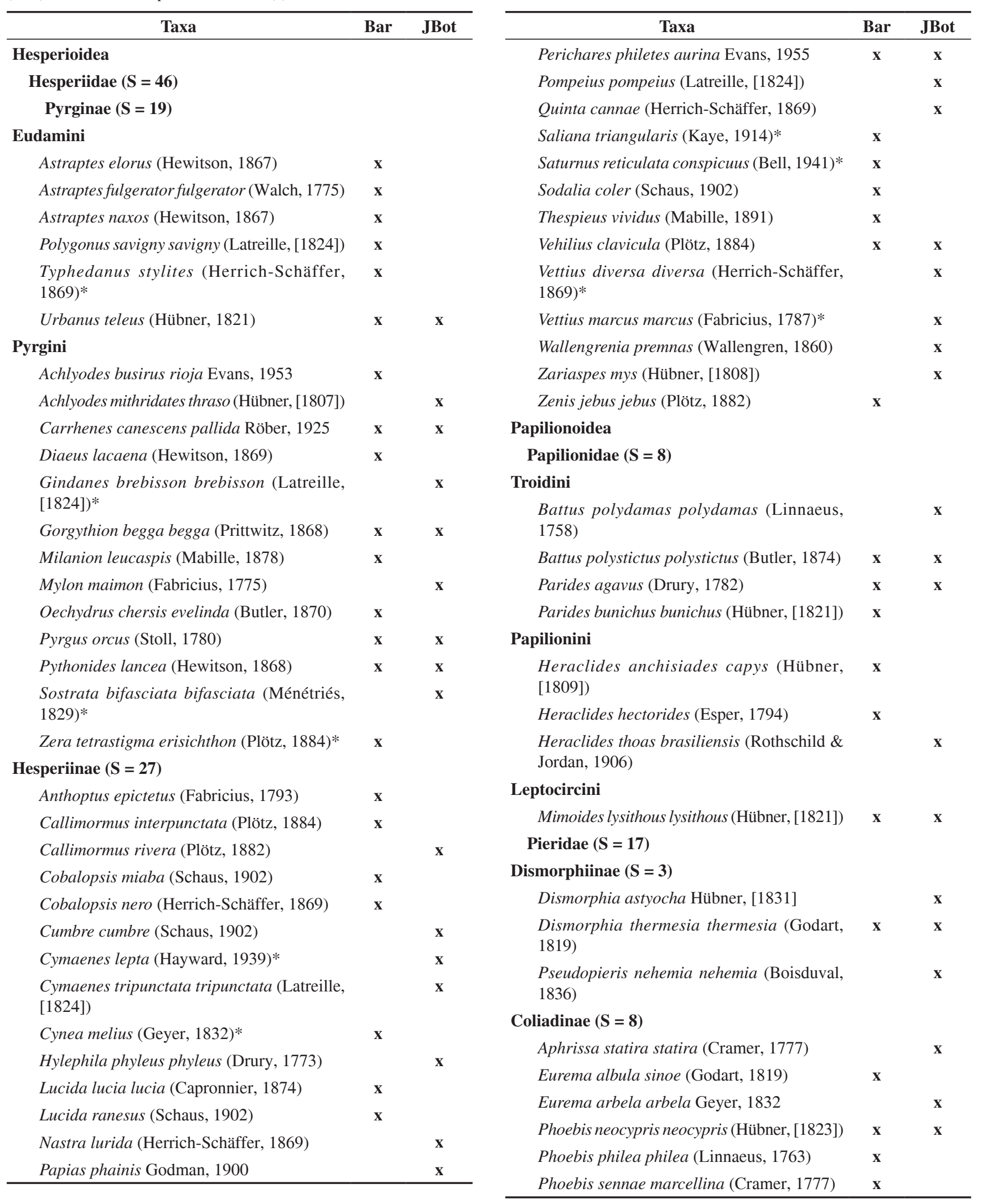


Tabela 1. Continuação...

\begin{tabular}{|c|c|c|}
\hline Taxa & Bar & JBot \\
\hline Pyrisitia leuce leuce (Boisduval, 1836) & $\mathbf{x}$ & $\mathbf{x}$ \\
\hline Rhabdodryas trite banksi (Breyer, 1939) & & $\mathbf{x}$ \\
\hline \multicolumn{3}{|l|}{ Pierinae $(S=6)$} \\
\hline \multicolumn{3}{|l|}{ Pierini } \\
\hline Catasticta bithys (Hübner, [1831]) & & $\mathbf{x}$ \\
\hline Leptophobia aripa balidia (Boisduval, 1836) & $\mathbf{x}$ & $\mathbf{x}$ \\
\hline Pereute swainsoni (Gray, 1832) & $\mathbf{x}$ & \\
\hline Tatochila autodice autodice (Hübner, 1818) & & $\mathbf{x}$ \\
\hline \multicolumn{3}{|l|}{ Anthocharidini } \\
\hline Hesperocharis erota (Lucas, 1852) & $\mathbf{x}$ & $\mathbf{x}$ \\
\hline Hesperocharis paranensis Schaus, 1898 & $\mathbf{x}$ & $\mathbf{x}$ \\
\hline \multicolumn{3}{|l|}{ Lycaenidae ( $S=13$ ) } \\
\hline \multicolumn{3}{|l|}{ Theclinae ( $\mathrm{S}=13$ ) } \\
\hline \multicolumn{3}{|l|}{ Eumaeini } \\
\hline Arawacus meliboeus (Fabricius, 1793) & $\mathbf{x}$ & $\mathbf{x}$ \\
\hline Atlides cosa (Hewitson, 1867) & & $\mathbf{x}$ \\
\hline Contrafacia imma (Pritwitz, 1865) & $\mathbf{x}$ & \\
\hline Cyanophrys acaste (Prittwitz, 1865)* & & $\mathbf{x}$ \\
\hline Erora sp. & & $\mathbf{x}$ \\
\hline Lamprospilus taminella (Schaus, 1902)* & $\mathbf{x}$ & \\
\hline Lamprosilus nubilum (H. H. Druce, 1907)* & & $\mathbf{x}$ \\
\hline Laothus phydela (Hewitson, 1867) & & $\mathbf{x}$ \\
\hline Magnastigma hirsuta (Prittwitz, 1865)* & & $\mathbf{x}$ \\
\hline Panthiades hebraeus (Hewitson, 1867)* & & $\mathbf{x}$ \\
\hline Parrhasius polibetes (Stoll, 1781)* & & $\mathbf{x}$ \\
\hline Parrhasius selika (Hewitson, 1874) & $\mathbf{x}$ & $\mathbf{x}$ \\
\hline Strephonota elika (Hewitson, 1867) & $\mathbf{x}$ & $\mathbf{x}$ \\
\hline Thepytus thyrea (Hewitson, 1867)* & & $\mathbf{x}$ \\
\hline \multicolumn{3}{|l|}{ Riodinidae (S = 7) } \\
\hline \multicolumn{3}{|l|}{ Riodininae $(S=7)$} \\
\hline \multicolumn{3}{|l|}{ Mesosemiini } \\
\hline Mesosemia odice (Godart, [1824]) & $\mathbf{x}$ & \\
\hline Ithomiola nepos (Fabricius, 1793) & $\mathbf{x}$ & \\
\hline \multicolumn{3}{|l|}{ Eurybiini } \\
\hline Eurybia pergaea (Geyer, 1832)* & $\mathbf{x}$ & \\
\hline \multicolumn{3}{|l|}{ Riodinini } \\
\hline Charis cadytis Hewitson, 1866 & $\mathbf{x}$ & \\
\hline Rhetus periander eleusinus Stichel, 1910* & $\mathbf{x}$ & $\mathbf{x}$ \\
\hline \multicolumn{3}{|l|}{ Tribo incertae sedis } \\
\hline Emesis russula Stichel, 1910 & $\mathbf{x}$ & \\
\hline \multicolumn{3}{|l|}{ Nymphidiini } \\
\hline Synargis regulus (Fabricius, 1793) & $\mathbf{x}$ & \\
\hline \multicolumn{3}{|l|}{ Nymphalidae ( $\mathrm{S}=75$ ) } \\
\hline \multicolumn{3}{|l|}{ Danainae $(S=2)$} \\
\hline \multicolumn{3}{|l|}{ Danaini } \\
\hline Danaus erippus (Cramer, 1775) & $\mathbf{x}$ & $\mathbf{x}$ \\
\hline
\end{tabular}

\begin{tabular}{llll}
\hline & Taxa & Bar & JBot \\
\hline Euploeini & &
\end{tabular}

Lycorea ilione ilione (Cramer, 1775) $\quad \mathbf{x}$

Ithomiinae $(\mathbf{S}=\mathbf{1 0})$

\section{Mechanitini}

Mechanitis lysimnia lysimnia (Fabricius, $\quad$ x $\quad$ x 1793)

Methona themisto (Hübner, 1818)

Thyridia psidii pallida Godman \& Salvin, $\quad \mathbf{x}$ 1898

\section{Napeogenini}

Epityches eupompe (Geyer, 1832) $\quad$ x $\quad$ x

Ithomiini

$\begin{array}{lll}\text { Ithomia drymo Hübner, } 1816 & \mathbf{x} & \mathbf{x}\end{array}$

Placidina euryanassa (C. Felder \& R. Felder, $\quad \mathbf{x} \quad \mathbf{x}$ 1860)

Dircenini

Dircenna dero dero (Hübner, 1823) $\quad$ x $\quad$ x

Episcada carcinia Schaus, $1902 \quad \mathbf{x} \quad \mathbf{x}$

Episcada hymenaea hymenaea (Prittwitz, $\quad \mathbf{x}$ 1865)

\section{Godyridini}

Pseudoscada erruca (Hewitson, 1855)

Morphinae $(\mathrm{S}=2)$

Morphini

Morpho anaxibia (Esper, [1801]) $\quad$ x $\quad$ x

Morpho portis thamyris (C. Felder \& R. $\quad \mathbf{x}$ Felder, 1867)

Brassolini

Caligo martia (Godart, [1824]) X

Satyrinae $(S=10)$

\section{Satyrini}

Eteona tisiphone (Boisduval, 1836)

Capronnieria galesus (Godart, [1824]) $\quad$ x

Godartiana muscosa (Butler, 1870) $\quad$ x

Hermeuptychia hermes (Fabricius, 1775) x

Moneuptychia griseldis (Weymer, 1911) $\quad$ x

Moneuptychia paeon (Godart, [1824]) X

Moneuptychia soter (Butler, 1877) $\quad$ x $\quad \mathbf{x}$

Paryphthimoides phronius (Godart, [1824]) $\quad$ x $\quad$ x

Taydebis peculiaris (Butler, 1874) $\quad$ x

Yphthimoides prox. viviana (Romieux, 1927)

Charaxinae $(\mathbf{S}=\mathbf{2})$

Anaeini

Memphis moruus stheno (Prittwitz, 1865) $\quad \mathbf{x}$

Zaretis itys itylus (Westwood, 1850)

$\mathbf{x} \quad \mathbf{x}$

Biblidinae $(S=14)$

\section{Biblidini}

Biblis hyperia nectanabis (Fruhstorfer, 1909) 
Tabela 1. Continuação...

\begin{tabular}{|c|c|c|}
\hline Taxa & Bar & JBot \\
\hline Cybdelis phaesyla (Hübner, [1831])* & & $\mathbf{x}$ \\
\hline $\begin{array}{l}\text { Diaethria candrena candrena (Godart, } \\
\text { [1824]) }\end{array}$ & $\mathbf{x}$ & $\mathbf{x}$ \\
\hline Diaethria eluina eluina (Hewitson, [1855]) & $\mathbf{x}$ & \\
\hline Dynamine agacles agacles (Dalman, 1823) & & $\mathbf{x}$ \\
\hline Dynamine myrrhina (Doubleday, 1849) & $\mathbf{x}$ & \\
\hline Ectima thecla thecla (Fabricius, 1796) & $\mathbf{x}$ & $\mathbf{x}$ \\
\hline Epiphile hubneri Hewitson, 1861 & $\mathbf{x}$ & $\mathbf{x}$ \\
\hline Epiphile orea orea (Hübner, [1823]) & $\mathbf{x}$ & $\mathbf{x}$ \\
\hline Eunica eburnea Fruhstorfer, 1907 & $\mathbf{x}$ & $\mathbf{x}$ \\
\hline $\begin{array}{l}\text { Hamadryas epinome (C. Felder \& R. Felder, } \\
\text { 1867) }\end{array}$ & $\mathbf{x}$ & \\
\hline Hamadryas februa februa (Hübner, [1823]) & & $\mathbf{x}$ \\
\hline Hamadryas fornax fornax (Hübner, [1823]) & $\mathbf{x}$ & \\
\hline Myscelia orsis (Drury, 1782) & $\mathbf{x}$ & \\
\hline \multicolumn{3}{|l|}{ Apaturinae $(S=2)$} \\
\hline Doxocopa kallina (Straudinger, 1886) & & $\mathbf{x}$ \\
\hline Doxocopa laurentia laurentia (Godart, [1824]) & $\mathbf{x}$ & $\mathbf{x}$ \\
\hline \multicolumn{3}{|l|}{ Nymphalinae ( $S=10)$} \\
\hline \multicolumn{3}{|l|}{ Nymphalini } \\
\hline Hypanartia bella (Fabricius, 1793) & $\mathbf{x}$ & \\
\hline Hypanartia lethe (Fabricius, 1793) & & $\mathbf{x}$ \\
\hline Vanessa braziliensis (Moore, 1883) & $\mathbf{x}$ & $\mathbf{x}$ \\
\hline \multicolumn{3}{|l|}{ Kallimini } \\
\hline Anartia amathea roeselia (Eschscholtz, 1821) & $\mathbf{x}$ & $\mathbf{x}$ \\
\hline Siproeta epaphus trayja Hübner, [1823] & $\mathbf{x}$ & \\
\hline \multicolumn{3}{|l|}{ Melitaeini } \\
\hline Eresia lansdorfi (Godart, 1819) & $\mathbf{x}$ & $\mathbf{x}$ \\
\hline Ortilia orthia (Hewitson, 1864) & $\mathbf{x}$ & $\mathbf{x}$ \\
\hline
\end{tabular}

circundado por bosques adjacentes (Figura 2) possui no seu interior reflorestamentos e áreas de acesso comum ao público. A combinação destes fatores pode estar influenciando na estruturação e composição da fauna de borboletas. Brown Junior \& Freitas (2002) em estudo de 15 parques da cidade de Campinas, concluíram que a conectividade é o fator ambiental que exerce maior influência sobre riqueza (neste caso é a composição) de borboletas.

$\mathrm{O}$ frio e as geadas dos meses do outono e inverno provocaram diminuição no número de exemplares amostrados, já que em determinadas amostragens foi registrada temperatura média de $12{ }^{\circ} \mathrm{C}$. Tal situação é comparável à ação dos ventos polares apresentado por Carneiro et al. (2008), que agem durante o inverno no sul da ilha de Santa Catarina, sendo considerado como um fator diretamente relacionado ao declínio da riqueza de borboletas durante os meses mais frios.

A comparação da riqueza deste estudo, com a apresentada por C. Mielke (1995), não é aplicável, uma vez que o estudo anterior utilizou principalmente dados de Coleções Entomológicas, o que tornou sua área de estudo ampla. Ainda no estudo de C. Mielke (1995) Hesperocharis paranensis Schaus, 1898 está mencionada

\begin{tabular}{|c|c|c|}
\hline Taxa & Bar & JBot \\
\hline Tegosa claudina (Eschscholtz, 1821) & $\mathbf{x}$ & $\mathbf{x}$ \\
\hline Tegosa orobia (Hewitson, 1864) & $\mathbf{x}$ & $\mathbf{x}$ \\
\hline Telenassa teletusa (Godart, [1824]) & $\mathbf{x}$ & $\mathbf{x}$ \\
\hline \multicolumn{3}{|l|}{ Limenitidinae $(S=8)$} \\
\hline Adelpha calliphane Fruhstorfer, 1915 & & $\mathbf{x}$ \\
\hline Adelpha falcipennis Fruhstorfer, $1915 *$ & $\mathbf{x}$ & $\mathbf{x}$ \\
\hline Adelpha gavina Fruhstorfer, 1915 & & $\mathbf{x}$ \\
\hline Adelpha hyas hyas (Doyère, [1840]) & $\mathbf{x}$ & \\
\hline Adelpha mythra (Godart, [1824]) & $\mathbf{x}$ & \\
\hline Adelpha serpa serpa (Boisduval, 1836)* & $\mathbf{x}$ & \\
\hline Adelpha syma (Godart, [1824]) & $\mathbf{x}$ & $\mathbf{x}$ \\
\hline Adelpha zea (Hewitson, 1850) & & $\mathbf{x}$ \\
\hline \multicolumn{3}{|l|}{ Heliconiinae ( $S=14)$} \\
\hline \multicolumn{3}{|l|}{ Acraeini } \\
\hline Actinote carycina Jordan, 1913 & $\mathbf{x}$ & $\mathbf{x}$ \\
\hline Actinote melanisans Oberthür, 1917 & $\mathbf{x}$ & $\mathbf{x}$ \\
\hline Actinote parapheles Jordan, 1913 & $\mathbf{x}$ & \\
\hline Actinote thalia pyrrha (Fabricius, 1775) & & $\mathbf{x}$ \\
\hline \multicolumn{3}{|l|}{ Heliconiini } \\
\hline Agraulis vanillae maculosa (Stichel, [1908]) & $\mathbf{x}$ & $\mathbf{x}$ \\
\hline Dione juno juno (Cramer, 1779) & & $\mathbf{x}$ \\
\hline Dryas iulia alcionea (Cramer, 1779) & $\mathbf{x}$ & $\mathbf{x}$ \\
\hline Eueides aliphera aliphera (Godart, 1819) & & $\mathbf{x}$ \\
\hline Eueides isabella dianasa (Hübner, [1806]) & & $\mathbf{x}$ \\
\hline Heliconius besckei Ménétriés, 1857 & $\mathbf{x}$ & $\mathbf{x}$ \\
\hline Heliconius erato phyllis (Fabricius, 1775) & $\mathbf{x}$ & $\mathbf{x}$ \\
\hline Heliconius ethila narcaea Godart, 1819 & $\mathbf{x}$ & $\mathbf{x}$ \\
\hline Heliconius sara apseudes (Hübner, [1813]) & $\mathbf{x}$ & \\
\hline Philaethria wernickei (Röber, 1906) & $\mathbf{x}$ & $\mathbf{x}$ \\
\hline
\end{tabular}

como Hesperocharis lactea lactea (Burmeister, 1879). Pode-se afirmar que a riqueza encontrada nos parques é considerável, visto que foram estudadas duas localidades, e a riqueza de espécies amostradas correspondeu a cerca de 1/3 (um terço) das espécies registradas para Curitiba por C. Mielke (1995), indicando que estas pequenas reservas urbanas, são de grande importância para conservação da fauna local de borboletas.

\section{Agradecimentos}

À Prefeitura Municipal de Curitiba, principalmente a Secretaria Municipal do Meio Ambiente, Departamento de Pesquisa e Monitoramento, pela licença de coleta e aos respectivos funcionários que prestativamente nos auxiliaram durante a realização do inventário.

Ao Instituto Tecnológico Simepar, em nome de Osmar Stringari e Edson Sakae Nagashima, pelos dados de temperatura e pluviométricos das datas de coleta.

E aos colegas, Msc. Diego Dolibaina, ao Msc. Eduardo Carneiro e ao Msc. Gustavo Cavalheiro Amarilia, pelo auxílio prestado durante a pesquisa. 


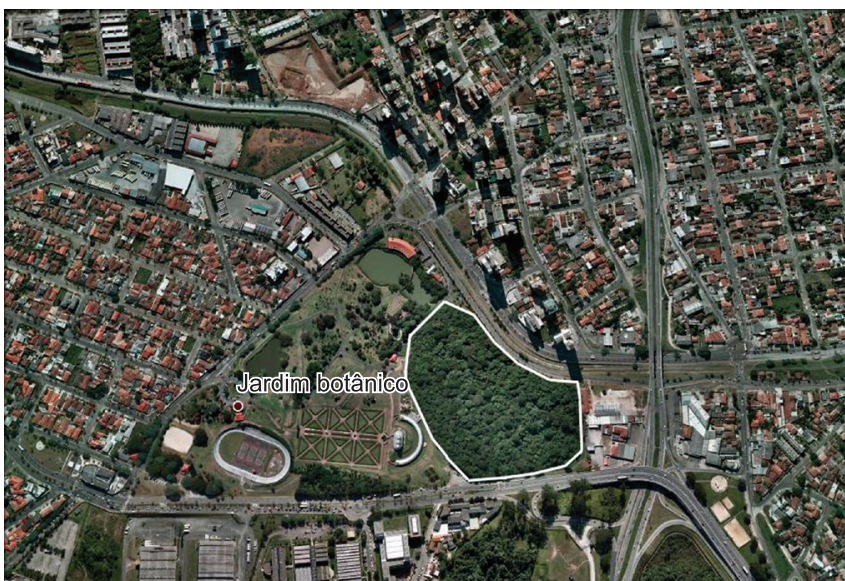

Figura 1. Jardim Botânico e área de estudo em destaque.

Figure 1. Botanical Garden and the study area highlighted.

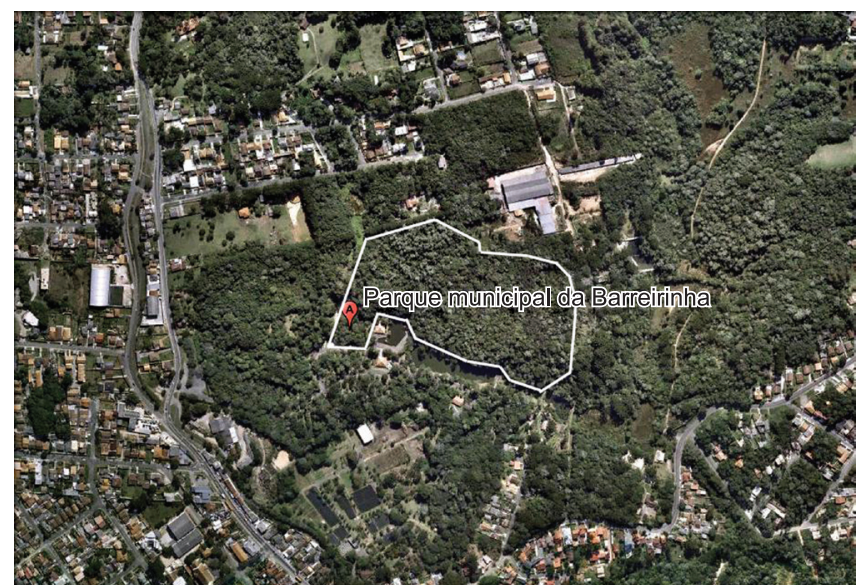

Figura 2. Parque Municipal da Barreirinha e área de estudo em destaque.

Figure 2. Municipal Park of Barreirinha and study area highlighted.

Tabela 2. Lista de famílias, subfamílias, tribos e espécies, número total de espécies, áreas de estudo, espécies exclusivas e Índice de Similaridade de Sorensen (IS) entre o Parque Barreirinha (Bar) e o Jardim Botânico (JBot).

Table 2. List of families, subfamilies, tribes and species, total species number, studied areas, unique species and Sorensen similarity index (SI) between the Municipal Park of Barreirinha (Bar) and the Botanical Garden (JBot).

\begin{tabular}{|c|c|c|c|c|c|c|}
\hline \multirow[t]{2}{*}{ Taxa } & \multicolumn{3}{|c|}{$\begin{array}{l}\text { Áreas de } \\
\text { estudo }\end{array}$} & \multicolumn{2}{|c|}{$\begin{array}{c}\text { Espécies } \\
\text { exclusivas }\end{array}$} & \multirow[b]{2}{*}{$\begin{array}{c}\text { Is } \\
(\%)\end{array}$} \\
\hline & $\begin{array}{l}\text { Espécies } \\
\text { totais }\end{array}$ & Bar & JBot & Bar & JBot & \\
\hline Hesperioidea & 46 & 29 & 24 & 22 & 17 & 26 \\
\hline Hesperiidae & 46 & 29 & 24 & 22 & 17 & 26 \\
\hline Pyrginae & 19 & 15 & 9 & 10 & 4 & 41 \\
\hline Eudamini & 6 & 6 & 1 & 5 & 0 & 28 \\
\hline Pyrgini & 13 & 9 & 8 & 5 & 4 & 47 \\
\hline Hesperiinae & 27 & 14 & 15 & 12 & 13 & 13 \\
\hline Papilionoidea & 120 & 85 & 83 & 37 & 36 & 55 \\
\hline Papilionidae & 8 & 6 & 5 & 3 & 3 & 36 \\
\hline Troidini & 4 & 3 & 3 & 1 & 1 & 66 \\
\hline Papilionini & 3 & 2 & 1 & 2 & 1 & 0 \\
\hline Leptocircini & 1 & 1 & 1 & 0 & 0 & 100 \\
\hline Pieridae & 17 & 10 & 13 & 4 & 7 & 52 \\
\hline Dismorphiinae & 3 & 1 & 3 & $\mathbf{0}$ & 2 & 50 \\
\hline Coliadinae & 8 & 5 & 5 & 3 & 3 & 40 \\
\hline Pierinae & 6 & 4 & 5 & 1 & 2 & 66 \\
\hline Pierini & 4 & 2 & 3 & 1 & 2 & 40 \\
\hline Anthocharidini & 2 & 2 & 2 & 0 & 0 & 100 \\
\hline Lycaenidae & 13 & 5 & 11 & 2 & 8 & 37 \\
\hline Theclinae & 13 & 5 & 11 & 2 & 8 & 37 \\
\hline Eumaeini & 13 & 5 & 11 & 2 & 8 & 37 \\
\hline Riodinidae & 7 & 7 & 1 & 6 & $\mathbf{0}$ & 25 \\
\hline Riodininae & 7 & 7 & 1 & 6 & $\mathbf{0}$ & 25 \\
\hline Mesosemiini & 2 & 2 & 0 & 2 & 0 & 0 \\
\hline Eurybiini & 1 & 1 & 0 & 1 & 0 & 0 \\
\hline Riodinini & 2 & 2 & 1 & 1 & 0 & 66 \\
\hline $\begin{array}{l}\text { Tribo incertae } \\
\text { sedis }\end{array}$ & 1 & 1 & 0 & 1 & 0 & 0 \\
\hline Nymphidiini & 1 & 1 & 0 & 1 & 0 & 0 \\
\hline
\end{tabular}

\begin{tabular}{|c|c|c|c|c|c|c|}
\hline \multirow[t]{2}{*}{ Taxa } & \multicolumn{3}{|c|}{$\begin{array}{l}\text { Áreas de } \\
\text { estudo }\end{array}$} & \multicolumn{2}{|c|}{$\begin{array}{c}\text { Espécies } \\
\text { exclusivas }\end{array}$} & \multirow[b]{2}{*}{$\begin{array}{c}\text { Is } \\
(\%)\end{array}$} \\
\hline & $\begin{array}{c}\text { Espécies } \\
\text { totais }\end{array}$ & Bar & JBot & Bar & JBot & \\
\hline Nymphalidae & 75 & 57 & 53 & 22 & 18 & 63 \\
\hline Danainae & 2 & 2 & 1 & 1 & $\mathbf{0}$ & 66 \\
\hline Danaini & 1 & 1 & 1 & 0 & 0 & 100 \\
\hline Euploeini & 1 & 1 & 0 & 1 & 0 & 0 \\
\hline Ithominae & 10 & 7 & 9 & 1 & 3 & 75 \\
\hline Mechanitini & 3 & 2 & 2 & 1 & 1 & 50 \\
\hline Napeogenini & 1 & 1 & 1 & 0 & 0 & 100 \\
\hline Ithomiini & 2 & 2 & 2 & 0 & 0 & 100 \\
\hline Dircennini & 3 & 2 & 3 & 0 & 1 & 80 \\
\hline Godyridini & 1 & 0 & 1 & 0 & 1 & 0 \\
\hline Morphinae & 3 & 3 & 1 & 2 & $\mathbf{0}$ & 50 \\
\hline Morphini & 2 & 2 & 1 & 1 & 0 & 66 \\
\hline Brassolini & 1 & 1 & 0 & 1 & 0 & 0 \\
\hline Satyrinae & 10 & 8 & 5 & 5 & 2 & 46 \\
\hline Satyrini & 10 & 8 & 5 & 5 & 2 & 46 \\
\hline Charaxinae & 2 & 2 & 1 & 1 & $\mathbf{0}$ & 66 \\
\hline Anaeini & 2 & 2 & 1 & 1 & 0 & 66 \\
\hline Biblidinae & 14 & 10 & 9 & 5 & 4 & 52 \\
\hline Biblidini & 14 & 10 & 9 & 5 & 4 & 52 \\
\hline Apaturinae & 2 & 1 & 2 & $\mathbf{0}$ & 1 & 66 \\
\hline Nymphahinae & 10 & 9 & 8 & 2 & 1 & 82 \\
\hline Nymphalini & 3 & 2 & 2 & 1 & 1 & 50 \\
\hline Kallimini & 2 & 2 & 1 & 1 & 0 & 66 \\
\hline Melitaeini & 5 & 5 & 5 & 0 & 0 & 100 \\
\hline Limenitidinae & 8 & 5 & 5 & 3 & 3 & 40 \\
\hline Limenitidini & 8 & 5 & 5 & 3 & 3 & 40 \\
\hline Heliconiinae & 14 & 10 & 12 & 2 & 4 & 72 \\
\hline Acraeini & 4 & 3 & 3 & 1 & 1 & 66 \\
\hline Heliconiini & 10 & 7 & 9 & 1 & 3 & 75 \\
\hline Total & 166 & 114 & 107 & 59 & 53 & 48 \\
\hline
\end{tabular}




\section{Referências bibliográficas}

BIENZANKO, C.M. 1938. Sobre alguns lepidópteros que ocorrem em arredores de Curitiba (Estado do Paraná). Livraria do Globo, Pelotas, 8 p.

BONFANTTI, D., DI MARE, R. A. \& GIOVENARDI, R. 2009. Butterflies (Lepidoptera: Papilionoidea and Hesperioidea) from two Forest fragments in northern Rio Grande do Sul, Brazil. Check List 5(4):819-829.

BROWN JUNIOR, K.S. 1992. Borboletas da Serra do Japi: diversidade, habitat, recursos alimentares e variação temporal. In História natural da Serra do Japi: ecologia e preservação de uma área florestal no sudeste do Brasil (L.P.C. Morellato, ed.). Unicamp/Fapesp, Campinas, p.142-187.

BROWN JUNIOR, K.S. \& FREITAS, A.V.L. 2002. Butterfly communities of urban forest fragments in Campinas, São Paulo, Brazil: Structure, instability, environmental correlates, and conservation. J. Insect Conserv. 6:217-231. http://dx.doi.org/10.1023/A:1024462523826

CARNEIRO, E., MIELKE, O.H.H. \& CASAGRANDE, M.M. 2008 Borboletas do sul da ilha de Santa Catarina, Florianópolis, Santa Catarina, Brasil (Lepidoptera: Hesperioidea e Papilionoidea). Shilap - Rev. Lepidopt. 36(142):261-271.

INSTITUTO DE PESQUISA E PLANEJAMENTO URBANO DE CURITIBA - IPPUC. http://www.ippuc.org.br (último acesso em $18 / 03 / 2010)$

KRISTENSEN, N.P., SCOBLE, M.J. \& KARSHOLT, O. 2007. Lepidoptera phylogeny and systematics: the state of inventorying moth and butterfly diversity. Zootaxa 1668:699-747.
LAMAS, G. 2004. Checklist: Part 4A. Hesperioidea - Papilionoidea. In Atlas of Neotropical Lepidoptera 5A (J.B. Heppner, ed.). Association of Tropical Lepidoptera, Gainesville.

MIELKE, C.G.C. 1995. Papilionoidea e Hesperioidea (Lepidoptera) de Curitiba e seus arredores, Paraná, Brasil, com notas taxonômicas sobre Hesperiidae. Rev. Bras. Zool. 11(4):759-776. http://dx.doi.org/10.1590/ S0101-81751994000400018

NEW, T.R. \& SANDS, D.P.A. 2002. Conservation concerns for butterflies in urban areas of Australia. J. Insect Conserv. 6:207-215. http://dx.doi. org/10.1023/A:1024425515889

RUSZCZYK, A. 1986a. Ecologia urbana de borboletas, I. O gradiente de urbanização e a fauna de Porto Alegre, RS. Revta. Brasil. Biol. 46(4):675688. http://dx.doi.org/10.1590/S0034-71081999000400007

RUSZCZYK, A. 1986b. Ecologia urbana de borboletas, II. Papilionidae, Pieridae e Nymphalidae em Porto Alegre, RS. Revta. Brasil. Biol. 46(4):689-706.

RUSZCZYK, A. 1987. Distribution and abundance of butterflies in the urbanization zones of Porto Alegre, Brazil. J. Res. Lepid. 25:157-78.

SHAPIRO, A.M. 2002. The Californian urban butterflies fauna is dependent on alien plants. Divers. Distr. 8(1):31-40. http://dx.doi.org/10.1046/j.13669516.2001.00120.x

SILVA, A.R.M., LANDA, G.G. \& VITALINO, R.F. 2007. Borboletas (Lepidoptera) de um fragmento de mata urbano em Minas Gerais, Brasil. Lundiana 8(2):137-142. 